\title{
HIGH-RESOLUTION SATELLITE IMAGING OF THE 2004 TRANSIT OF VENUS AND ASYMMETRIES IN THE CYTHEREAN ATMOSPHERE
}

\author{
Jay M. Pasachoff ${ }^{1,2}$, Glenn Schneider ${ }^{3}$, and Thomas Widemann ${ }^{4}$ \\ ${ }^{1}$ Williams College-Hopkins Observatory, 33 Lab Campus Drive, Williamstown, MA 01267, USA \\ ${ }^{2}$ Department of Planetary Science, California Institute of Technology, 150-21 1200 East California Boulevard, Pasadena, CA 91125, USA \\ ${ }^{3}$ Steward Observatory, The University of Arizona, 933 North Cherry Avenue, Tucson, AZ 85721, USA \\ ${ }^{4}$ Observatoire de Paris-LESIA, 92190 Meudon, France \\ Received 2010 November 1; accepted 2010 December 2; published 2011 March 1
}

\begin{abstract}
This paper presents the only space-borne optical-imaging observations of the 2004 June 8 transit of Venus, the first such transit visible from Earth since AD 1882. The high-resolution, high-cadence satellite images we arranged from NASA's Transition Region and Coronal Explorer (TRACE) reveal the onset of visibility of Venus's atmosphere and give further information about the black-drop effect, whose causes we previously demonstrated from TRACE observations of a transit of Mercury. The atmosphere is gradually revealed before second contact and after third contact, resulting from the changing depth of atmospheric layers refracting the photospheric surface into the observer's direction. We use Venus Express observations to relate the atmospheric arcs seen during the transit to the atmospheric structure of Venus. Finally, we relate the transit images to current and future exoplanet observations, providing a sort of ground truth showing an analog in our solar system to effects observable only with light curves in other solar systems with the Kepler and CoRoT missions and ground-based exoplanet-transit observations.
\end{abstract}

Key words: history and philosophy of astronomy - occultations - planets and satellites: atmospheres - planets and satellites: general - planets and satellites: individual (Venus)

Online-only material: color figure, animations, supplementary data (FITS) files (tar.gz)

\section{INTRODUCTION}

Transits of Venus have been observed from Earth only in $1639,1761,1769,1874$, and 1882 prior to 21st century's 2004 and 2012 transits. Historically, transits of Venus were expected to provide measurement of the astronomical unit. The method was proposed after the Mars-parallax successful observation by Cassini, Richer, and Picard from Paris and Cayenne in 1672. Halley (1716) suggested that the distance to the Sun could be directly obtained by the observation of a transit of Venus from distant stations on Earth, making these international expeditions integral to arguably the most important problem in astronomy for over two centuries, since Kepler's law provided only proportional spacings of the planets (e.g., Woolf 1959; van Helden 1995; Chapman 1998; Ferguson 1999; Maunder \& Moore 1999; Maor 2000; Hughes 2004; Sellers 2001; Pasachoff \& Filippenko 2007).

We have provided a history of prior observations of transits of Venus in Schneider et al. (2001, 2004, 2005) and Pasachoff et al. (2003, 2005a), in which we used spacecraft observations of the 1999 transit of Mercury to solve the long-standing problem of the black-drop effect, showing its origin in both a pointspread function and the extreme solar limb darkening at the extreme limb. We also discussed the history of the black-drop effect during transits of Venus in Pasachoff et al. (2003, 2005a); though our ground-based observations were from Greece, to be able to observe both black drops with favorable weather predictions, our paper appeared in the proceedings of the IAU Symposium (Kurtz \& Bromage 2005) that was held during the 2004 transit at the site in England at which Jeremiah Horrocks had, in 1639, first observed a transit of Venus (Horrocks 1639, as cited in Hevelius 1662).

Our series of observations of transits of Mercury in 1999 and 2003 and, later, of Venus in 2004 had been motivated by Schaefer
(2000, 2001), who had pointed out that more than half the recent published mentions of the black-drop effect were incorrect in discussing its cause, mistakenly attributing the effect to Venus's atmosphere, which is too narrow to provide the observed effect. Motivated also by the historical accounts of previously observed transits of Venus and our previous work on the black-drop effect (Pasachoff et al. 2005a), we took advantage of the first transit of Venus to be seen from Earth since the ascending node pair of 1874 and 1882, which accounted for both black-drop and special atmospheric effects (Figure 1).

Beside revisiting those long-standing issues resulting from the extreme scarcity of transit events (a couple of events, spaced by $8 \mathrm{yr}$, every 121.5 then $105.5 \mathrm{yr}$ ), we also desired to use the 2004 June 8 event as a surrogate to test observing methods, strategies, and techniques which have since been implemented by the CoRoT and Kepler space missions to detect and characterize extrasolar terrestrial planets as they transit their host stars.

We have previously reported (Schneider et al. 2005, 2006) on the detection of the 2004 transit of Venus in the Sun's total solar irradiance as an $0.1 \%$ dip lasting hours, with its ingress, egress, and mid-transit variations providing a direct analog to the exoplanet transit observations, though with much higher accuracy and detail (Schneider \& Pasachoff 2006; Pasachoff et al. 2008).

It is usually stated that Venus's atmosphere was discovered by Lomonosov at Saint-Petersburg's Observatory on 1761 May $26($ O.S.) $=$ June 6 during the descending node transit couple of 1761-1769 (Marov 2005). Lomonosov reported an aureole of light on the planet's limb, which he related to an atmosphere around the planet. Sheehan (2011, private communication) notes that Lomonosov's actual wording seems to correspond better to a black drop than to a Cytherean atmosphere and credits Johann Schröter in the 1790s for first firmly establishing the existence of Venus's atmosphere. Atmospheric effects have been 


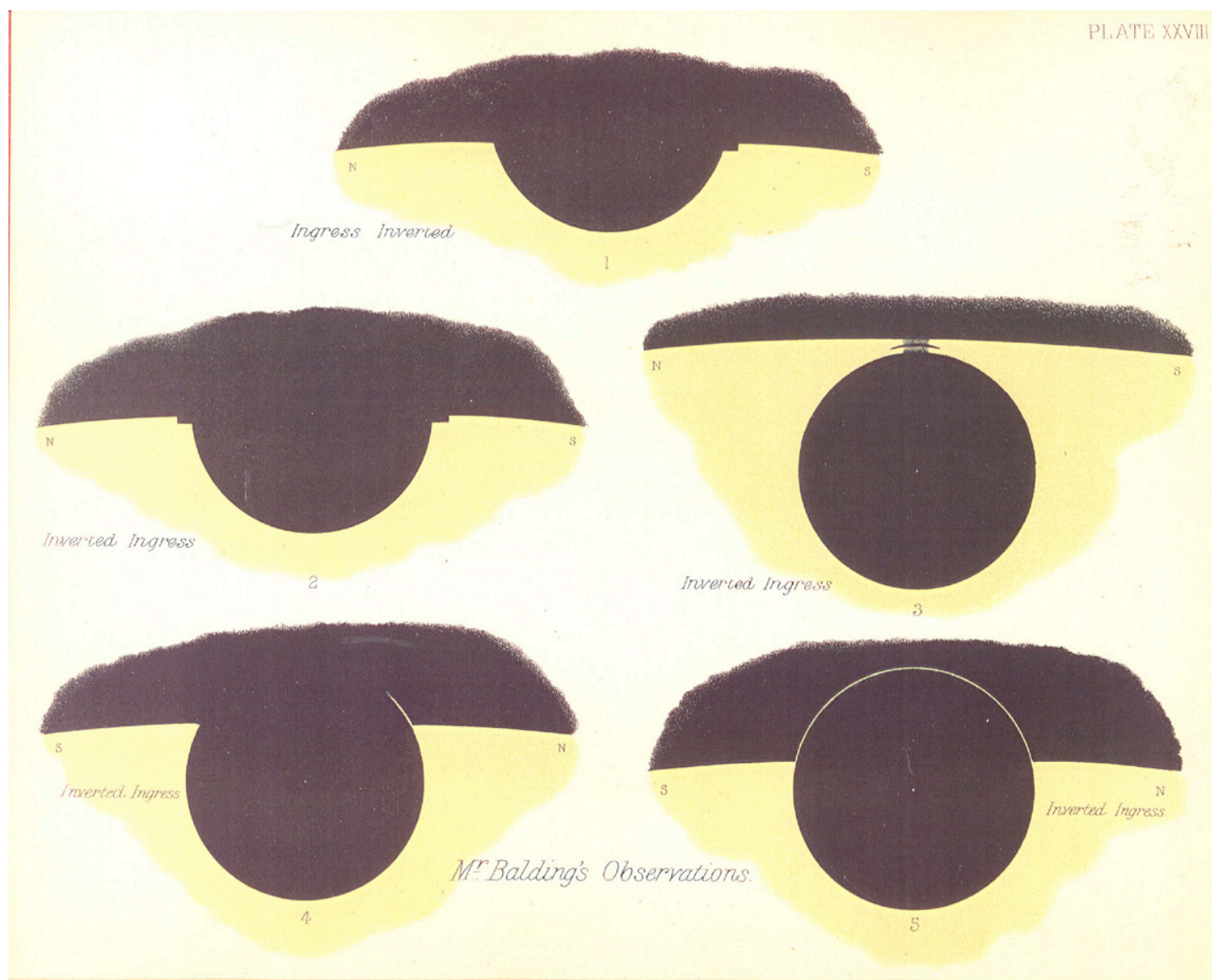

Figure 1. Egress sequence during the 1874 transit of Venus (east sky is on the right). It was observed from Sydney and reported by the Australian government astronomer (H. C. Russell 1875). The series of drawings shows the Cytherean atmosphere coming into view after third contact, along the Venus limb opposed to the Sun. An increased, persistent concentration of light refracted from the Sun is seen in Venus's northern polar atmosphere as indicated by H. C. Russell on the two last drawings. The black-drop effect is represented on the third image, at third contact; see Section 3.2 for a qualitative interpretation of the phenomena.

(A color version of this figure is available in the online journal.)

subsequently detected at various, non-transit Venus inferior conjunctions in the crescent's cusps (H. N. Russell 1899). Historical reviews are provided, e.g., by Link (1969), Tanga (2004), and Sheehan \& Westfall (2004).

\section{OBSERVATIONS}

With the advantage of contemporary fast photometry instrumental techniques, our primary programmatic objectives for the 2004 transit were three fold: using spatially resolved Transition Region and Coronal Explorer (TRACE) images to revisit previously described black-drop and atmospheric aureole effects on one part, using the spatially unresolved Active Cavity Radiometer Irradiance Monitor (ACRIM) instrument on board ACRIMsat for the solar irradiance (Pasachoff et al. 2005b; Schneider et al. 2006).

1. Black drop: we intended to use the space-based imagery to demonstrate that the-little-cited-conjecture that groundbased historical reports of "black drops" (e.g., Bergman 1761 and Figure 1; see Pasachoff et al. 2005a) at innerplanet transits are in fact a result of convolution of the instrumental (telescopic/optical) with atmospheric pointspread function in conjunction with the limb-darkened photospheric illumination function, as we showed was the case to explain the black-drop effect for the 1999 transit of Mercury (see Schneider et al. 2004);
2. Refraction of solar light: our second purpose was to obtain the first, space-borne fast-cadence images of the circum-Cytherian "aureole," which results from refraction of sunlight in the observer's direction by layers of the upper atmosphere of Venus, with high spatial resolution and image stability, with the purpose of providing a throughout brightness latitudinal distribution as a function of time, prior to contact II and after contact III. This aureole results from refraction of sunlight in the observer's direction, by layers of the upper atmosphere of Venus. This objective has acquired a renewed interest in the context of the Venus Express orbiter investigation of the upper atmosphere structure of Venus in the polar region (García Muñoz et al. 2009; Ignatiev et al. 2009; Widemann et al. 2010a, 2010b).

3. Testbed for photometry of an exoplanet transit: in a separate study using the un-spatially resolved ACRIM instrument, we followed the effect of the transit of Venus on the total solar irradiance and related it to current and future exoplanet observations, providing a sort of ground truth showing an analog in our solar system to effects observable only with light curves in other solar systems with the Kepler and CoRoT missions and ground-based exoplanet-transit observations (Schneider et al. 2005b).

In order to meet the science objectives (1) and (2) requiring imagery at high spatial resolution, we used NASA's TRACE spacecraft (Golub \& Pasachoff 2009) to image the 2004 June 
8 transit of Venus across the undiscriminated visible spectrum from UV to near-IR (see Section 2.1), at very high temporal cadence during (and flanking) the planet's crossing of the solar limb and while on the heavily limb-darkened portion of the solar disk (see Section 2.3). We simultaneously observed in Greece (Pasachoff et al. 2004b) and coordinated with observations with the Swedish 1-m Solar Telescope on La Palma in the Canary Islands, Spain (Pasachoff et al. 2006), in order to supplement the space-borne study with ground-based measurements (Pasachoff 2004).

\subsection{TRACE Wavelengths}

All images for this program were obtained in TRACE's "white light" (WL) configuration, which provides spectral sensitivity across the UV-visible and near-IR wavelength range from 1200 to $9600 \AA$. We therefore reproduced quite well spectrally the experimental conditions of previous centuries various observing reports. The TRACE WL instrumental response to an un-limb-darkened solar spectral energy distribution, peaking 6000-8000 A, is shown in Figure 4 of Schneider et al. (2004). Details appear in Handy et al. (1999).

\subsection{Spatial Scale and Resolution}

During the 2004 transit, Venus's angular diameter was $\sim 58.2$ arcsec $(12104 \mathrm{~km}$ at $0.289 \mathrm{AU})$, diametrically spanning 116 detector pixels. The TRACE WL spatial resolution is Nyquist limited by its $0^{\prime \prime} .5$ per pixel sampling of its CCD, not diffraction limited by the $30 \mathrm{~cm}$ telescope. With a WL pivot wavelength of $6200 \AA$, Venus was resolved by $\sim 60$ bandintegrated resolution elements.

\subsection{Temporal Cadence}

With a desire for high inter-frame temporal cadence in highest fidelity data format (i.e., "lossless" or uncompressed), particularly during and near the limb contacts, we read out only a $512 \times 512$ sub-array of the $1024 \times 1024$ CCD detector due to both downlink bandwidth and memory (image buffer) limitations. As our observations were interleaved with observations taken for other programs (with other TRACE filters and data formats; DeForest et al. 2009), the inter-frame temporal spacing was not uniform throughout our imaging sequences. During ingress, we achieved a cadence of five frames per minute (uniformly spaced) across the limb contacts, but at a reduced rate of $\sim$ two frames per minute at other times in order to minimize the volume of data over the course of the transit itself. At transit egress we obtained images at CIII $\sim \pm 3$ minutes at a (uniformly spaced) rate of seven frames per minute, but at a slower rate of one frame every $\sim 35 \mathrm{~s}$ when the leading edge of Venus was further from the solar limb. Some technical details of the as-planned observing program may be found, through http://www.transitofvenus.info along with other transit information, at http://nicmosis.as.arizona.edu:8000/ECLIPSE_WEB/ TRANSIT_04/TRACE/TOV_TRACE.html.

\subsection{Images, “Movies,” and Stereograms}

We present here the first photometrically rigorous results from the ingress and egress imaging sequence taken in TRACE's WL passband. The TRACE WL optical channel, so we discovered, suffers from a $\sim 1 \%$ intensity optical "ghosting." Normally, this ghosting is not of concern for TRACE's solar observation programs, but as our interest rests primarily in the "bottom" $1 \%$ of the (12 bit) dynamic sampling range, this ghosting presented a significant photometric "challenge." With detailed characterization we found the "ghost" to be double, with different degrees of afocality and intensity, plus an additional diffuse (not afocally specular) component more difficult to analytically characterize. For each frame, we built and subtracted models of the instrumentally scattered light to remove these artifacts (examples of this will be added later). The images presented here have been post-processed in this manner, and while not "perfect," this processing very effectively mitigates the instrumentally ghosted/ scattered light. This procedure, however, cannot recover intensity information with sufficient photometric precision in a $\sim 2$ pixel $\left(1^{\prime \prime} .0-1^{\prime \prime} .5\right)$ wide region exterior to the photosphere where the solar limb is bifurcated by the Cytherian disk. This is the reason for the "gap" you will note in the image of Venus presented here as Venus transits the solar limb.

In addition to the images presented here, movies and threedimensional (3D) stereograms of the transit made from selected TRACE imaging frames are available through the link http://www.transitofvenus.info.

The ingress sequence (Figures 2 and 3) is comprised of 98 frames from 050553 UT to 054751 UT, optimized for the atmosphere and for any black drop, respectively. The egress sequence (Figures 4 and 5) is comprised of 84 frames from 105231 UT to 112315 UT, optimized for the atmosphere and for any black drop, respectively. Both sequences have been assembled into movies with a playback rate of 10 frames per second. The movies (ingress atmosphere and black drop optimized and egress atmosphere and black drop optimized) are rendered as 16 bit (32 kilobyte-level gray scale). These animations track on the Sun; a third version for each ingress and egress has been computed to track on Venus's disk. Especially for astronomers at the times of the 2117 and 2124 transits, we are also providing the ingress and egress images in FITS formats as supplemental material.

\section{IMAGE PROCESSING AND RESULTS}

In the first six frames of the ingress sequence and in the "Venus Atmospheric Refraction" movies, a linear dynamic display range is used, from -15 (hard black) to +55 (hard white) instrumental counts (DN: data numbers) per pixel. (TRACE has a dynamic data range of 4096 DN.) The background sky has been median normalized to zero $\mathrm{DN}$, and the $1 \sigma$ noise in the background is $\sim 2 \mathrm{DN}$. In the bottom two frames of the ingress sequence, and in the "No Intrinsic Black Drop" movies, a display stretch over a larger dynamic range was made, to better define the limb of Venus against the "noise" of the solar granulation. The same two display stretches are used for the egress sequence images.

The solar photospheric radial limb-darkening profile has been removed from each of these images. This "flattens" the intensity of the photosphere and collapses the dynamic display range, thus very well shows the photospheric granulation. After "flattening," the intensity variation in the photospheric features is 20 times greater than the light seen, at its brightest, in the Cytherean atmospheric aureole.

\subsection{Optimizing the Image Dynamic Display Range for the Black-drop Effect}

In the image sequence frames from the "No Intrinsic Black Drop" movies, the photosphere has been attenuated only by an additional factor of two, after the removal of the solar limb darkening. Venus obscures the portion of the solar limb 


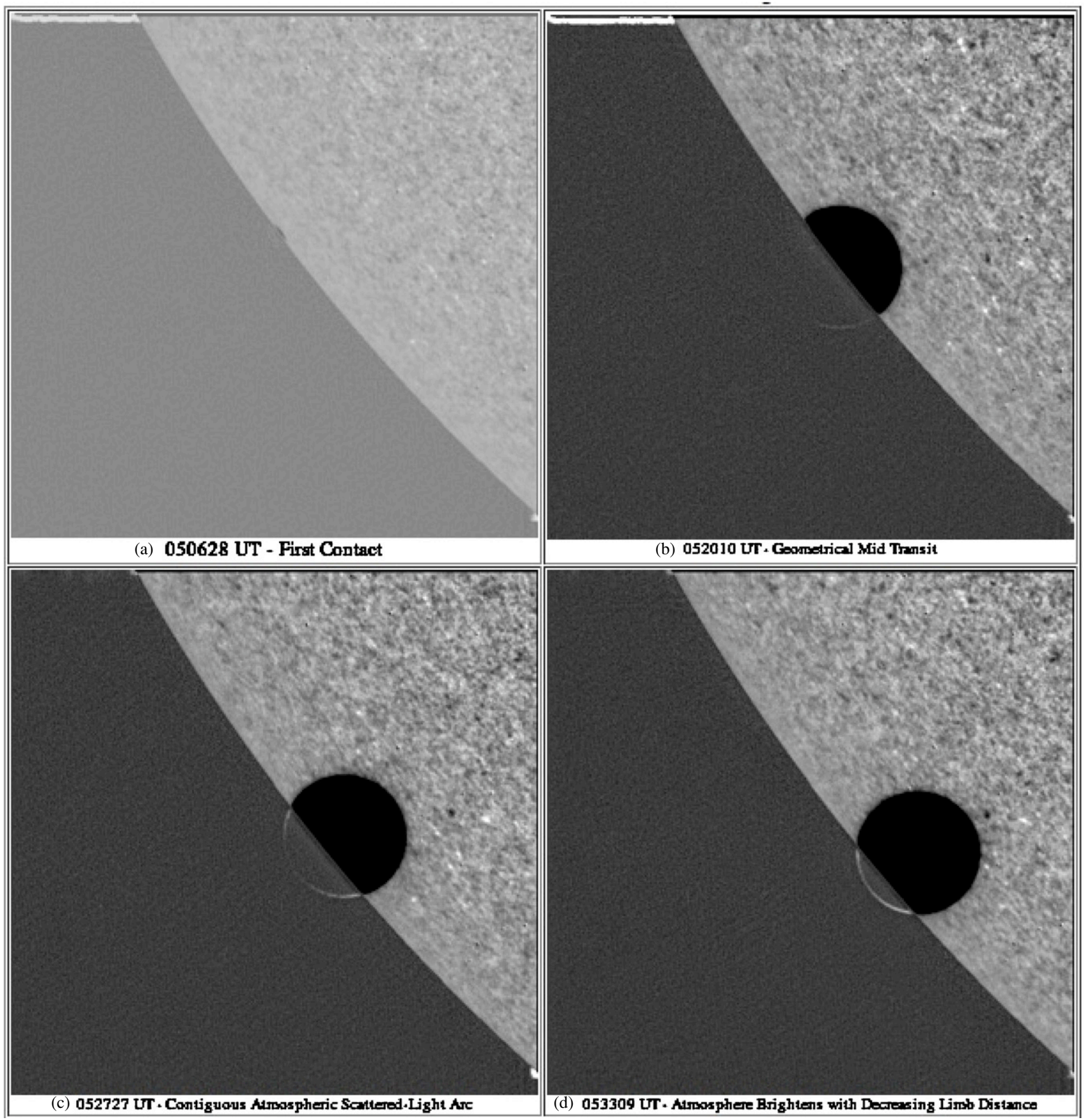

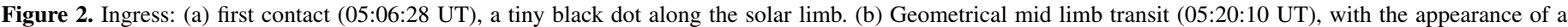

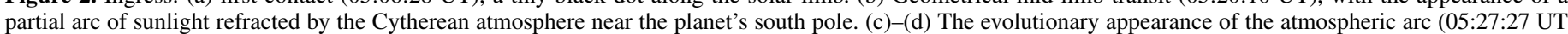

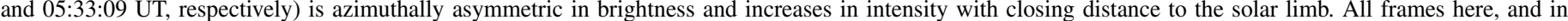
Figures 3-5, are oriented with north up and east to the left.

between contacts I and II and contacts III and IV. As a guide to the eye, during those time intervals, the location of the limb "behind" Venus is annotated with a thin arc at the photosphere/sky background interface. The "geometric" midingress is not coincident in time with temporal mid-transit, due to the parallactic motion of Venus induced by TRACE's Sunsynchronous orbit about Earth.

\subsection{Optimizing the Imaging Dynamic Display Range for Limb Atmospheric Refraction}

To simultaneously visualize Venus's atmosphere against the sky, and Venus's limb against the photosphere, the solar disk (with Venus in front of it) has been reduced/scaled in intensity by a factor of 10 . In Figures 2-5, the median level of the photosphere-after flattening by removing the radial limbdarkening profile (see Schneider et al. 2004; cf. Section 2.2 and Figures 7-9) and subsequent reduction in intensity by a factor of 10 - has been set to $+25 \mathrm{DN}$ (rather than zero, i.e., offset from the sky) to enable, very easily, seeing the solar limb against the sky background. With this display stretch, the interior silhouetted disk of Venus appears hard black against the granular photosphere (i.e., similar to the contrast as it appears to the eye with the Sun appropriately filtered). Simultaneously, the disk of Venus against the sky appears as 

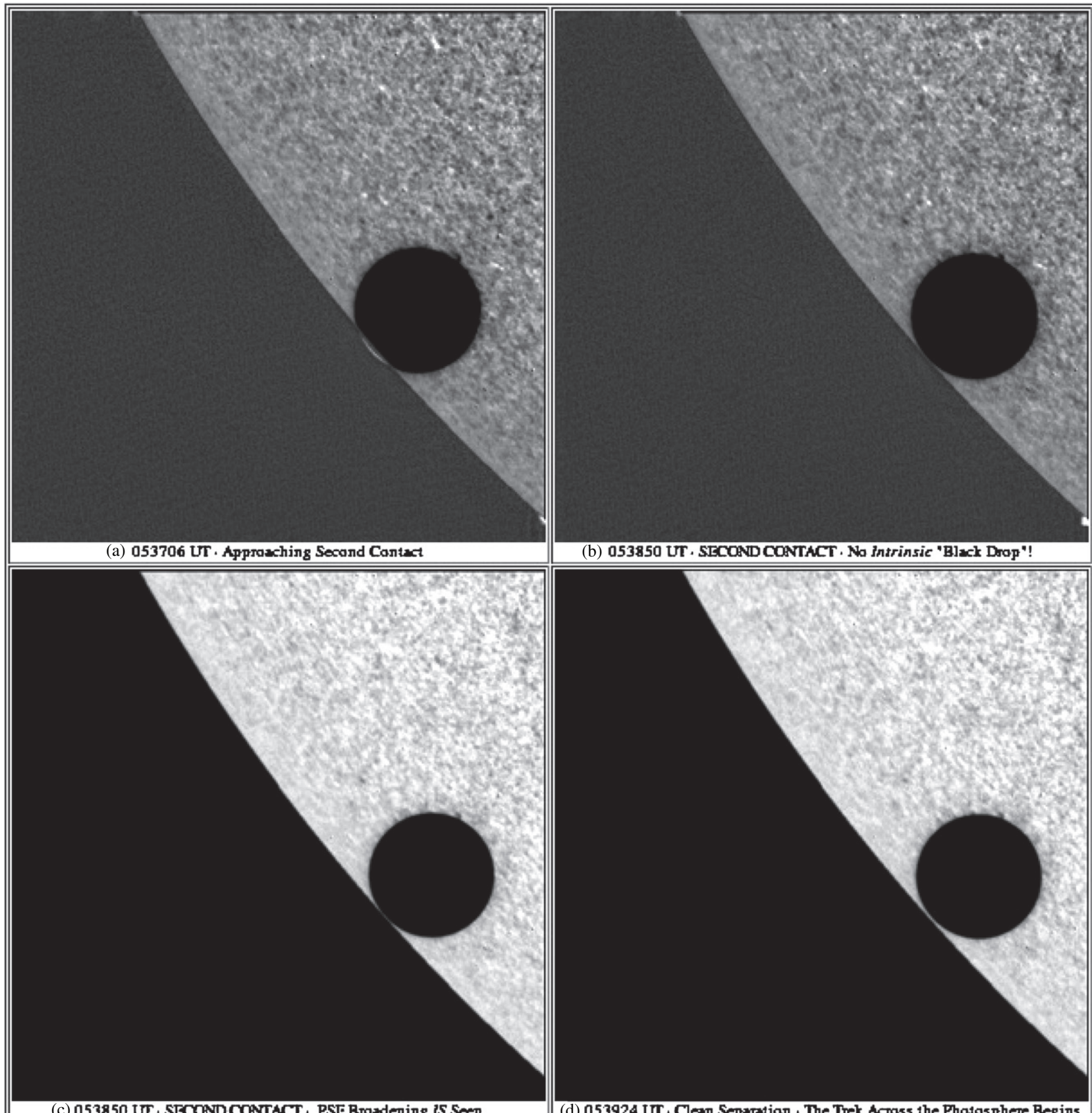

(b) 053850 UT, SECCOND CONTACT · Na Intrinsic "B]wek Drop"!

(c) 053850 UT , SIECCNDCCNTACT . PST Brawdening IS Seen

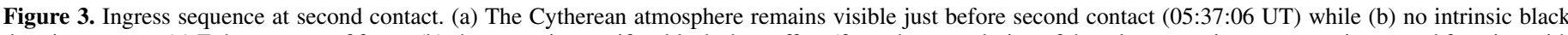

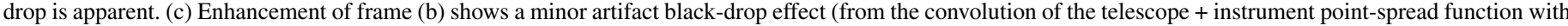

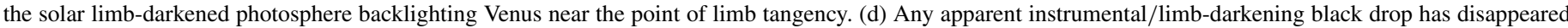

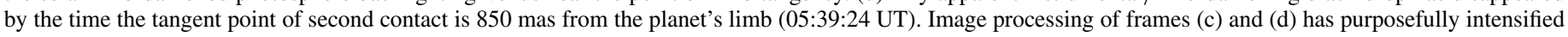
the section of the image outside the solar limb with respect to the solar photosphere; see the text for details.

(Animations and FITS files of this figure are available in the online journal).

the same intensity as the sky, which as Venus is backlit, is appropriate.

The TRACE images very clearly show the emergence of sunlight (refracted) by the Cytherean atmosphere as Venus approaches the line of sight to the solar disk. The phenomenon is clearly asymmetric, as a small arc of atmosphere first becomes visible on the southern disk of Venus, near the Venus south pole, not on a line perpendicular to the solar limb. By "geometrical mid-ingress," when the solar limb is equally bifurcated by Venus, this arc of scattered light is readily apparent.
Stereograms showing the phenomena in 3D appear in Figure 6; higher-resolution versions are available in the online edition.

About 12 minutes before second contact the atmospheric arc becomes "full" and contiguous, and grows brighter with decreasing angular distance to the solar limb. Upon egress the asymmetry in the brightness along the atmospheric arc is striking. Second (053850 UT image) and third (115849 UT image) contacts are geometrically "sharp" except for "blurring" by the 1" full-width at-half-maximum TRACE WL point-spread 


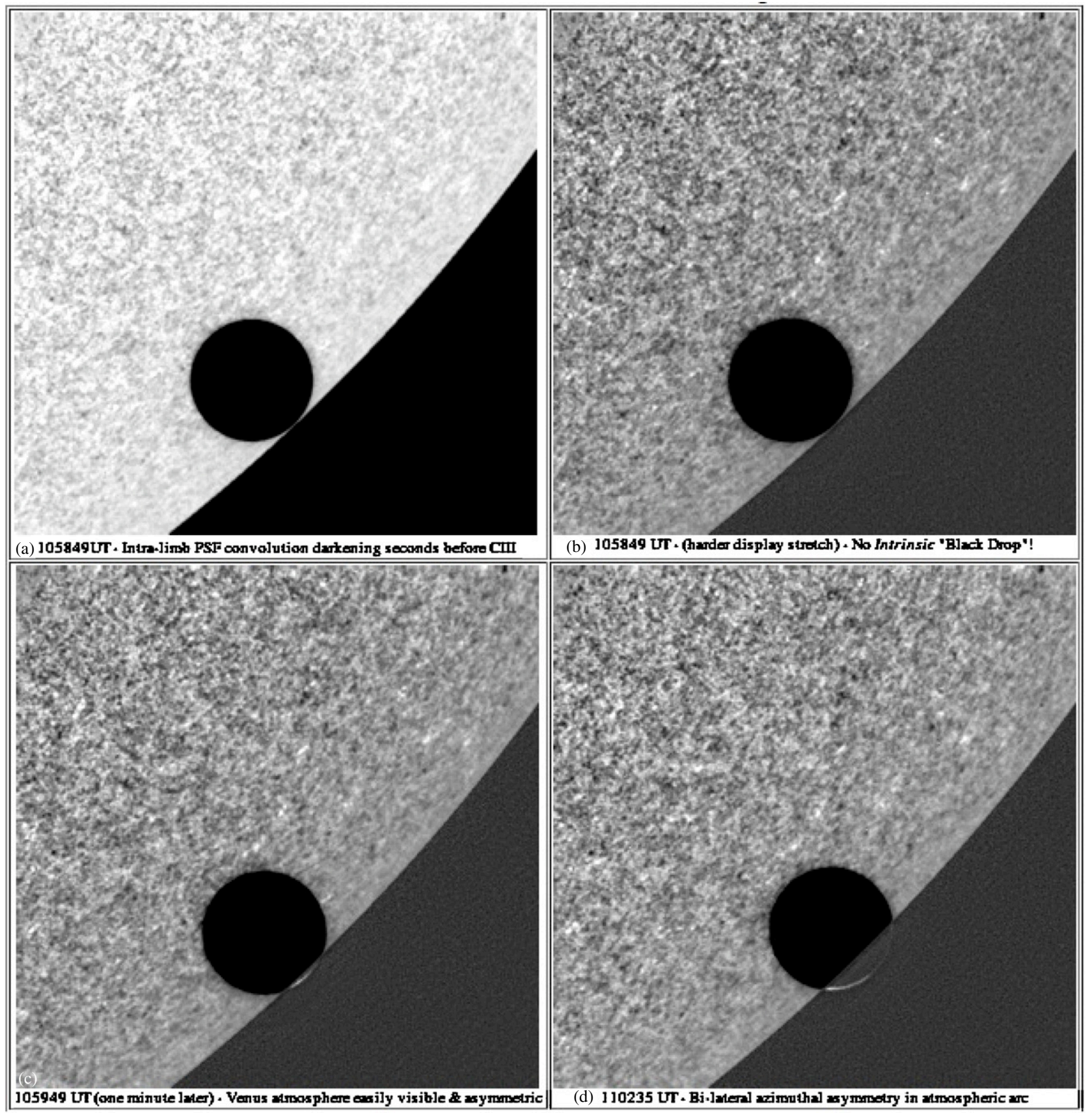

Figure 4. Egress. (a) Third contact (10:58:49 UT). (b) As in Figure 3(c), a minor black-drop of instrumental + limb-darkening origin is seen in contrast-enhanced frame (b) (same data as frame (a)). (c) First visibility of Venus's atmosphere after the contact (10:59:49 UT). (d) The brightness asymmetry along the Cytherean atmospheric arc in, and flanking, the southern polar region after third contact (11:02:35 UT) is similar to what was reported by Russell for the 1874 transit (see Figure 1; Link 1969; Tanga 2004). The observed aureole results from the refraction of solar photospheric emission by the limb upper atmosphere of Venus. The asymmetry results from latitudinal differences in atmospheric refractive properties (see Section 3.2).

(Animations and FITS files of this figure are available in the online journal).

function and residual instrumentally scattered light in the TRACE satellite optical system.

\section{DISCUSSION AND CONCLUSIONS}

Our spatially resolved TRACE observations obtained during the 2004 transit of Venus, as illustrated by Figures 3(c) and (d) and 4(a), show no evidence of the classical/historical "black-drop" effect. This finding illustrates the success for the predicted negative result based on TRACE WL observations of the 1999 November 15 transit of Mercury (Schneider et al. 2004;
Pasachoff et al. 2005a), where it has been demonstrated that the effect for both Mercury and Venus inner-planet transits was a result of the convolution of the instrumental (telescopic/optical) with the atmospheric point-spread function in conjunction with the limb-darkened photospheric illumination function. The centuries-old effect is therefore a non-intrinsic phenomenon (it is gone, now, the way of the Martian canals). Since for a transit of Mercury, the telescope's point-spread function alone was insufficient to explain the black-drop effect, and since we could explain the entire effect only by convolving the point-spread 


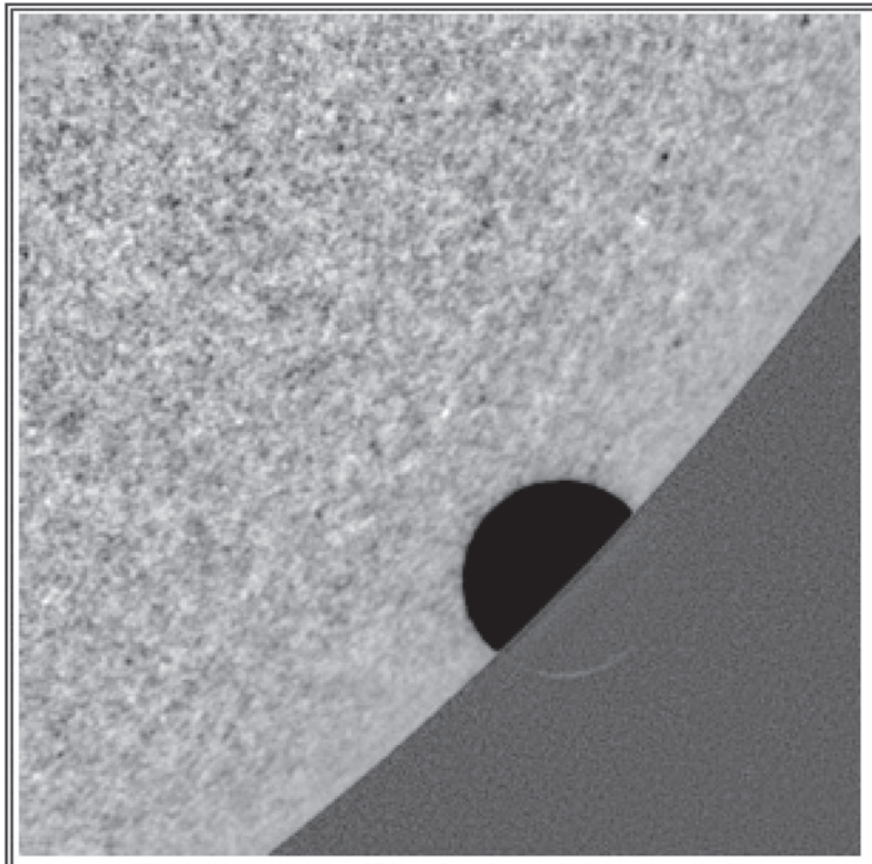

(a) 1 10823 UT, . Halfway thraugh egres, half the anc Jemsins

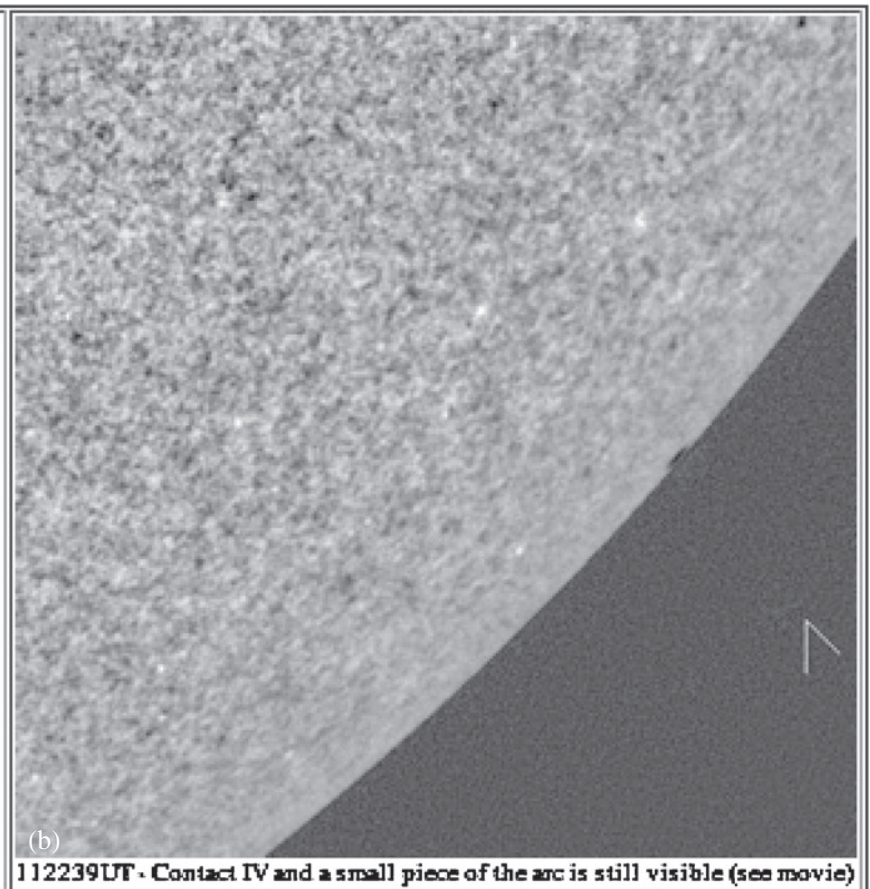

1 12239UT . Confact TV and a small piene of the arc is still visible (see movie)

Figure 5. Egress. (a) At geometrical mid-egress (11:08:23 UT), about half of the illuminated arc of Venus's atmosphere remains visible. With the gradual disappearance of Venus's illuminated atmosphere, refracted solar radiation at diminished intensity asymmetrically persists along the atmospheric arc extending $\sim 30^{\circ}$ in latitude from the Cytherean south pole. This atmosphere region is dynamically enclosed in a bulge of cold air (polar collar) and where the Venus cloud tops are lower by $7-10 \mathrm{~km}$ than at lower latitudes (see Section 4). (b) Seconds before fourth contact (11:22:39 UT), with only a tiny bit of Venus's dark disk silhouetted against the solar photosphere at the point of limb tangency, a small, faint sliver of the atmospheric arc near the south pole remains visible (location marked with an arrow).

function with the sharply varying limb-darkening profile at the extreme solar limb (Schneider et al. 2004), there is every reason to extrapolate that result to transits of Venus.

The dry, $96.5 \%$ carbon dioxide $\left(\mathrm{CO}_{2}\right)$ and $3.5 \%$ nitrogen $\left(\mathrm{N}_{2}\right)$ atmosphere of Venus has been extensively surveyed by both orbiter and in situ measurements in the last three decades. Its study has been the subject of pioneering solar system exploration-Venus was the destination of the first interplanetary spacecraft, Venera-1, launched by the Soviets on 1961 February 12. Although the atmosphere was first claimed by Lomonosov in the 1700 s, and $\mathrm{CO}_{2}$ discovered in it by Adams \& Dunham (1932), its 4-5 day superrotation was still debated in the 1970s and only fully recognized after the highly successful Pioneer Venus orbiter and multi-probe mission (1979-1992).

In situ measurements have been performed essentially in the mesosphere below $100 \mathrm{~km}$ and below the clouds. Information about minor atmospheric constituents, their concentration, reactions, sources, and sinks remains essentially incomplete, as only scarce measurements of localized vertical profiles have been performed above $100 \mathrm{~km}$ altitude, where temperature fields, especially in the polar regions, as well as their time variability, are still debated (see, e.g., Vandaele et al. 2008; Clancy et al. 2008). The upper cloud structure itself reveals distinct dynamical structure in the latitudinal direction, with convective, wave-dominated zonal flow in the lower latitude range near Venus's equator, and a significant transition to less convective banded flow between $45^{\circ}$ up to about $\pm 70^{\circ}-80^{\circ}$. Ground-based Doppler wind velocities confirm the mean zonal flow deduced from tracking of cloud features (Widemann et al. 2007, 2008; Widemann \& Liao 2010). The temperature field forms a bulge of cold air at $60^{\circ}-80^{\circ}$ latitude called the "cold collar region." This torus-like structure encloses a vast vortex-type structure several thousand kilometers across, with a slower rotation period (from
-2.5 to -2.8 Earth days) and significantly depressed cloud top altitude, at about $62-65 \mathrm{~km}$ instead of 70-74 km closer to the equator (Svedhem et al. 2007; Ignatiev et al. 2009).

Simulation of the transit circumstances reveals that the tangent point at second contact was about $25^{\circ}$ from the Cytherean equator and the tangent point at third contact was at latitude approximately $45^{\circ}$. Initial modeling of the effect of differential refraction on the deviation of solar light by an isothermal atmosphere, of scale height about 3.5-4 km (e.g., García Muñoz et al. 2009), in the direction of the observer indicates the rays originating from the solar photosphere cross a layer about 35-40 km above cloud tops (P. Tanga 2011, private communication). This geometrical property of the upper atmosphere is in agreement with the latitudinal increase of refracted sunlight in the transit aureole we imaged using the TRACE WL data and especially visible in Figures 2(b), 4(d), and 5(a) of this paper. The latitudinal extent on Venus south polar limb matches very well the polar vortex region southward of $70^{\circ}-80^{\circ}$, where cloud tops are lower by about $8-10 \mathrm{~km}$. Similar agreement can be traced back to Russell's drawings of 1874 (Figure 1) for the northern hemisphere of Venus.

The arc of atmosphere seen includes the polar region of Venus and indicates that the asymmetry is due to the latitudinal dependence of upper cloud altitude northward of the polar collar in the polar vortex region of Venus's atmosphere. Additional constraints on Venus's latitudinal distribution of upper cloud altitude from the ESA Venus Express mission are being compared with ground-based observations and with our TRACE spacebased observations by Tanga et al. (2011). We have set up a collaboration with Tanga to measure brightness of the atmospheric arc as functions of position along the arc and of time for both the 2004 observations and, one hopes, eventually the 2012 observations. 


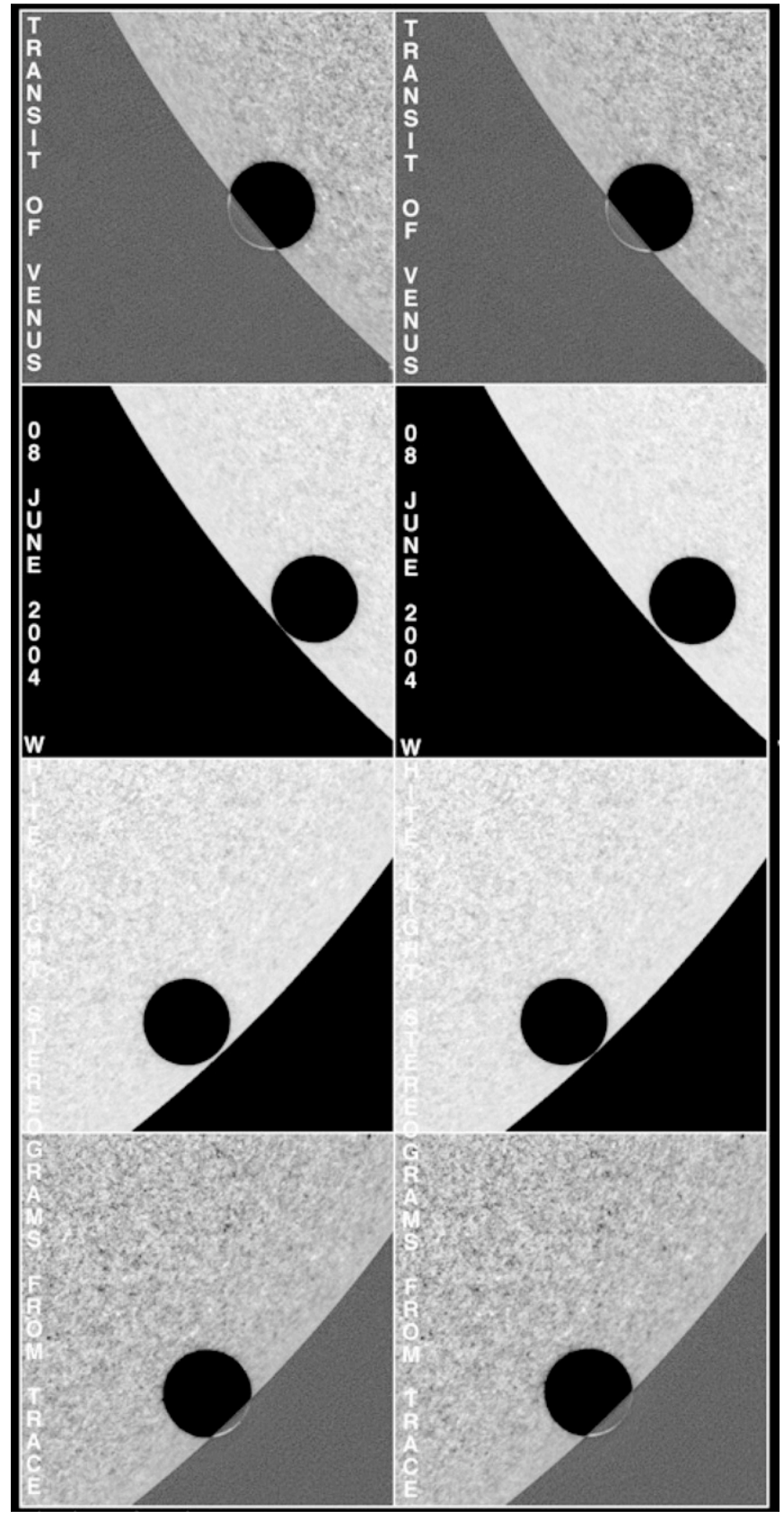

Figure 6. Stereograms, with pairs of images taken over short intervals of time. The stereo effect can be seen by most people by simply letting the eyes relax. The $3 \mathrm{D}$ effect is more profound in the high-resolution images that are available in the online edition. They can be viewed from a larger distance, which is more relaxing for the eyes.

Regarding the photometry of the event compared to the total solar irradiance, using the spatially unresolved Venus transit light curve obtained by ACRIMsat (and a similar one obtained by SORCE/TIM) by Schneider et al. (2006), variations were found to be $0.0018 \%$ at the $1 \sigma$ level (compared to a $0.0019 \%$ expected change in signal) with inter-region variations in internal dispersions of $0.00022 \%$. Hence, a sensitivity to the presence versus the absence of a Venus-like opaque planetary atmosphere was tested at only a $1.05 \sigma$ level of confidence, allowing the authors to discuss the ability to detect exoplanet stellar transits photometrically with scalably comparable instrumental sensitivities. Because newer generation programs to detect super-Earths and their atmospheric signature by exoplanet primary transits are currently submitted as future programs, our team has started an investigation of intermediate-spectral-resolution techniques to probe the molecular species content of the refractive layers near $110 \mathrm{~km}(80 \mathrm{~km})$ in the polar vortex region by positioning a spectro-imaging device in the ingress and egress region from a solar observatory, without a coronographic system. This procedure would eventually lead us to more quantitative assessments on $\mathrm{CO}_{2}$ broadband detection below $190 \mathrm{~nm}$ in the aureole spectrum, as well as upper limits of detectable species in the context, including the $\mathrm{CO}$ rovibration $2-0$ band at $2.3 \mu \mathrm{m}$, and the $1-0$ band at $4.7 \mu \mathrm{m}$.

Now that the Atmospheric Imaging Assembly on NASA's Solar Dynamics Observer (Golub \& Pasachoff 2009) has replaced TRACE, its $12 \mathrm{~s}$ cadence for eight of its 10 filters and full-disk field of view with similar 0.5 arcsec pixels meaning that the transit of Venus observations on 2012 June 6 will be automatically made, without the need for the special arrangements that we made for TRACE cadence and pointing in 2004. Venus Express's and other observations of Cytherean clouds will be used for explaining any observed transit asymmetries for the 2012 transit, our last opportunity to do so from Earth until the next pair of transits of Venus, which will be in the ascending node, on 2117 December 11 and 2125 December 8.

We thank Karel Schrijver, Ted Tarbell, and other members of the TRACE science and operations teams for their invaluable assistance and efforts in planning and implementing these observations; and Leon Golub at the Harvard-Smithsonian Center for Astrophysics for advice on TRACE observations and data. Kayla Gaydosh (then at Bryn Mawr College) assisted with the image calibration and post-processing under a summer research program supported by the Keck Northeast Astronomy Consortium. David Butts, Joseph Gangestad, and Owen Westbrook also assisted on site in Thessaloniki, Greece, with our coordinated ground-based observations. We thank John Seiradakis of the Aristotle University of Thessaloniki for his collaboration. We also thank the Committee for Research and Exploration of the National Geographic Society for their researchgrant support for the transit of Venus expedition. Pasachoff's solar observations were supported in part by Guest Investigator NASA grant NNG04GK44G for work with the TRACE spacecraft and are now supported in part by NASA Marshall grant NNX10AK47A. Pasachoff's planetary work is supported in part by grant NNX08AO50G from NASA Planetary Astronomy to Williams College. He thanks Michael Brown and the Department of Planetary Science at Caltech for hospitality during the completion of this paper.

The Transition Region and Coronal Explorer, TRACE, is a mission of the Stanford-Lockheed Institute for Space Research (a joint program of the Lockheed-Martin Advanced Technology Center's Solar and Astrophysics Laboratory and Stanford's Solar Observatories Group), and part of NASA's Small Explorer program.

\section{REFERENCES}

Adams, W. S., \& Dunham, T. 1932, PASP, 44, 243

Bergman, T. 1761, Phil. Trans., 52, 227

Chapman, A. 1998, Endeavour, 22, 148

Clancy, R. T., Sandor, B. J., \& Moriarty-Schieven, G. H. 2008, Planet. Space i., 56, 1344

DeForest, C. E., Martens, P. C. H., \& Wills-Davey, J. M. 2009, ApJ, 690, 1264 Ferguson, K. 1999, Measuring the Universe (New York: Walker)

García-Muñoz, A., Mills, F. P., Slanger, T. G., Piccioni, G., \& Drossart, P. 2009, J. Geophys. Res., 114, E12002 
Golub, L., \& Pasachoff, J. M. 2009, The Solar Corona (2nd ed.; Cambridge: Cambridge Univ. Press)

Halley, E. 1716, Phil. Trans., 29, 454

Handy, B. N., et al. 1999, Sol. Phys., 187, 229

Hevelius, J. 1662, Mercurius in Sole Visus Gedani, anno Christiano MDCLXI. . . cui annexa est Venus in sole pariter visa, anno 1639... Liverpoliae, a Jeremia Horroxio, nunc primum edita. Simon Reiniger for the author. Danzig

Hughes, D. W. 2004, in IAU Colloq. 196, Transits of Venus: New Views of the Solar System and Galaxy, ed. D. W. Kurtz \& G. E. Bromage (Cambridge: Cambridge Univ. Press)

Ignatiev, N. I., et al. 2009, J. Geophys. Res., 114, E00B43

Kurtz, D. W., \& Bromage, G. E. (ed.) 2005, in IAU Colloq. 196, Transits of Venus: New Views of the Solar System and Galaxy (Cambridge: Cambridge Univ. Press)

Link, F. 1969, Eclipse Phenomena in Astronomy (Berlin: Springer)

Maor, E. 2000, June 8, 2004-Venus in Transit (Princeton, NJ: Princeton Univ. Press)

Marov, M. Y. 2005, in IAU Colloq. 196, Transits of Venus: New Views of the Solar System and Galaxy, ed. D. W. Kurtz \& G. E. Bromage (Cambridge: Cambridge Univ. Press)

Maunder, M., \& Moore, P. 1999, Transit: When Planets Cross the Sun (Berlin: Springer)

Pasachoff, J. M. 2004, Sky Telesc., 108, 82

Pasachoff, J. M., \& Filippenko, A. 2007, The Cosmos: Astronomy in the New Millennium (3rd ed.; Boston, MA: Cengage)

Pasachoff, J. M., Schneider, G., \& Golub, L. 2003, in IAU 01082, Joint Discussion on Mercury, 25th Sydney IAU General Assembly, ed. O. Engvold (Paris: IAU), 156, 1204

Pasachoff, J. M., Schneider, G., \& Golub, L. 2005a, in IAU Colloq. 196, Transits of Venus: New Views of the Solar System and Galaxy, ed. D. W. Kurtz \& G. E. Bromage (Cambridge: Cambridge Univ. Press)

Pasachoff, J. M., Schneider, G., \& Kiselman, D. 2006, Working Group on the Transits of Venus, 26th IAU General Assembly, Prague, ed. K. A. van der Hucht (Paris: IAU), 17

Pasachoff, J. M., Schneider, G., \& Willson, R. C. 2005b, BAAS, 36, 1566

Pasachoff, J. M., Schneider, G., \& Willson, R. C. 2008, BAAS, 40, 404
Pasachoff, J. M., et al. 2004, BAAS, 36, 1161

Russell, H. C. 1875, The Transit of Venus (Sydney: Sydney Observatory)

Russell, H. N. 1899, ApJ, 9, 284

Schaefer, B. E. 2000, BAAS, 332, 1383

Schaefer, B. E. 2001, J. Hist. Astron., 32, 325

Schneider, G., \& Pasachoff, J. M. 2006, Working Group on the Transits of Venus, 26th IAU General Assembly, Prague, ed. K. A. van der Hucht (Paris: IAU), 17

Schneider, G., Pasachoff, J. M., \& Golub, L. 2001, BAAS, 33, 1037, http:// nicmosis.as.arizona.edu:8000/POSTERS/TOM1999.jpg

Schneider, G., Pasachoff, J. M., \& Golub, L. 2005, in Highlights of Astronomy 13, ed. O. Engvold (Cambridge: Cambridge Univ. Press), 70

Schneider, G., Pasachoff, J. M., \& Golub, L. 2004, Icarus, 168, 249

Schneider, G., Pasachoff, J. M., \& Willson, R. C. 2006, ApJ, 641, 565

Sheehan, W., \& Westfall, J. 2004, The Transits of Venus (Amherst, NY: Prometheus Press)

Sellers, D. 2001, The Transit of Venus-The Quest to Find the True Distance of the Sun (Leeds: MagdaVelda Press)

Svedhem, H., Titov, D., Taylor, F., \& Witasse, O. 2007, Nature, 450, 629

Tanga, P. 2004, http://www.eso.org/public/outreach/eduoff/vt-2004/Background/ Infol2/paolo-venustwilight

Tanga, P., et al. 2011, Icarus, submitted

Vandaele, A. C., et al. 2008, J. Geophys. Res., 113, E00B23

van Helden, A. 1995, in The General History of Astronomy. II: Planetary Astronomy from the Renaissance to the Rise of Astrophysics, ed. R. Taton \& C. Wilson (Cambridge: Cambridge Univ. Press), 153

Widemann, T., Lellouch, E., \& Campargue, A. 2007, Planet. Space Sci., 55, 1741

Widemann, T., Lellouch, E., \& Donati, J.-F. 2008, Planet. Space Sci., 56, 1320

Widemann, T., \& Liao, E. 2010, BAAS, 42, 974

Widemann, T., Machado, P., Liao, Y., \& Young, E. 2010a, in Venus 2010 International Workshop, ed. S. S. Limaye (Madison, WI: Univ. Wisconsin), http://venus.wisc.edu/workshop

Widemann, T., et al. 2010b, in International Venus Conference, Aussois, France, ed. P. Drossart (Paris: Obs. de Paris), http://lesia.obspm.fr/venus2010

Woolf, H. 1959, The Transits of Venus: A Study in Eighteenth Century Science (Princeton, NJ: Princeton Univ. Press) 